\title{
Identifying Depression in South Asian Patients with End-Stage Renal Disease: Considerations for Practice
}

\author{
Shivani Sharma Kamaldeep Bhui Joseph Chilcot \\ David Wellsted Ken Farrington
}

School of Psychology, University of Hertfordshire, Hatfield, UK

\section{Key Words}

Depression $\cdot$ End-stage renal disease $\cdot$ South Asians $\cdot$ Measurement $\cdot$ Screening

\begin{abstract}
Depression is a prevalent burden for patients with end-stage renal disease (ESRD) and one that is under-recognized and consequently under-treated. Although several studies have explored the association between depression symptoms, treatment adherence and outcomes in EuroAmerican patient groups, quantitative and qualitative exploration of these issues in patients from different cultural and ethnic backgrounds has been lacking. This review discusses the methodological issues associated with measuring depression in patients of South Asian origin who have a 3- to 5-fold greater risk of developing ESRD. There is a need to advance research into the development of accurate screening practices for this patient group, with an emphasis on studies utilizing rigorous approaches to evaluating the use of both emic (culture-specific) and etic (universal or culture-general) screening instruments.

Copyright $\odot 2011$ S. Karger AG, Basel
\end{abstract}

\section{Introduction}

End-stage renal disease (ESRD) is a growing public health problem [1] and one that has a significant impact on the lives of patients [2]. There are multiple sources of stress associated with the loss of kidney function and reliance on a multifaceted treatment regime. The reliance 
upon dialysis is one of these stressors, with other adjustments including changes in family roles, mobility, dietary constraints, medication effects, worry over the dependency on treatment and fear of death [3-5]. Furthermore, depression is a prevalent problem in patients with ESRD. The Diagnostic and Statistical Manual of Mental Disorders 4th edition (DSM-IV) defines major depressive disorder as the loss of interest or pleasure for weeks accompanied by a constellation of 5 or more psychological, behavioural and somatic symptoms, and classifies depression as a disease which is primarily generic or neurochemical in aetiology [6]. The symptoms of depression include depressed mood, sleep disturbances, fatigue, reoccurring thoughts of death or suicide and a lack of energy. In chronically ill populations, it is estimated that depression accounts for half of the identified psychopathology [7], which can lead to increased clinical and psychological morbidity due to reduced compliance with treatment and reduced functional levels [8]. Indeed, in ESRD, several studies have demonstrated the association between depression symptoms, quality of life (QoL) $[9,10]$ and survival [11-15].

Although the prevalence of depression in ESRD is largely unknown, its impact on treatment outcomes has made it critical to advance the psychological assessment of patients. The assessment of depression typically involves self-complete screening instruments and the use of clinical diagnostic interviews, either of which are administered according to diagnostic classification systems such as the DSM-IV or the International Classification of Diseases (ICD-10). Screening instruments typically employ cut-off scores which can be used to characterize a patient's symptoms as mild, moderate or severe in terms of depressive symptomatology. In chronically ill populations, the accuracy of screening instruments is, however, limited due to 'criterion contamination', which is the overlap of somatic symptoms associated with the underlying medical illness and depressive symptomatology $[2,8]$. Whether or not screening and diagnostic schemes should remove overlapping symptoms is a topic of much debate, and there is limited evidence to suggest that the cognitive and somatic symptoms associated with depression can be separated from affective and behavioural components [16-18]. Nonetheless, several studies in the renal field have now reported on the clinical diagnostic accuracy of screening instruments such as the Beck Depression Inventory (BDI-II [19]) for this patient group, finding that the prevalence of depression in ESRD ranges from 20 to $30 \%$ and indeed contributes to adverse clinical outcomes, although precise measurement remains a pertinent issue [2].

The advances made in the clinical understanding of depression symptoms in ESRD will, however, benefit some patients more than others. Research in this area has, in the main, focused on the experiences and development of screening practices for patients from 'white Euro-American' backgrounds, which remains a significant issue in terms of generalizability. This may be because there are unique barriers that prevent clinicians from exploring the concerns and symptoms of patients from other cultural and ethnic groups. The aim of this article is to discuss the issues surrounding screening and diagnosis of depression in South Asian patients (patients originating from the Indian subcontinent including India, Pakistan, Bangladesh and Sri Lanka) with ESRD.

\section{Prevalence of ESRD in South Asians}

Whilst ESRD is a recognized health problem across the world, its prevalence in South Asian populations is unknown because of insufficient national and international registries and poor government policies to support patients from developing countries [20]. It is however clear that within the United Kingdom (UK), there is a significantly higher incidence of ESRD in South Asians [21,22]. It is estimated that South Asians are 3 to 5 times more likely to develop ESRD when compared to white Europeans [23-26]. This has often been explained 
in terms of the greater prevalence of diabetes in South Asians, which is the most common cause of ESRD [27], making this group more vulnerable to diabetic nephropathy than other minority and European groups, often despite uniform medical care [25, 28, 29]. Consequently, there is a high demand for renal transplants amongst South Asians in the UK, although the supply of suitable kidneys is low, as is the rate of transplantation [30]. It is however encouraging that studies conducted in both the UK and the United States report that survival rates in ESRD are not compromised due to ethnicity. In fact, several studies have reported better survival rates in South Asian dialysis patients [31-34]. Although the determinants of better survival in South Asians are not fully understood, factors such as higher mean Kt/V have been associated with more favourable outcomes [34].

\section{Depression in South Asian Patients with ESRD: Challenges for Accurate Screening}

To our knowledge, there are no published studies on the prevalence of depression in haemodialysis patients of South Asian origin. A study carried out with patients receiving peritoneal dialysis in India reported that $65 \%$ of patients experienced some depressive symptoms, and that $19 \%$ of patients had severe depressive symptomatology [35]. The dearth of research into the prevalence of depression in this patient group is coupled with few qualitative investigations into the attitudes, thoughts and feelings of South Asian patients toward renal care, which makes it difficult to understand the range of factors that impact treatment adherence and outcomes, and to make selected comparisons with other groups. There is however evidence to suggest that QoL, which is an important propensity of patient outcomes, is significantly lower in South Asian patients receiving renal replacement therapy when compared to their caregivers [36], and other minority and white Euro-American patients [37]. Specifically, South Asian patients are more likely to view ESRD as a social burden, even when successfully treated by transplantation [37]. Differences in QoL appear to be unrelated to treatment modality, treatment adequacy and comorbidities [37]. So whilst South Asian patients may not be disadvantaged in treatment outcomes, as suggested by quantitative investigations, there may be differences in patient experiences that impact psychological wellbeing - an area that has been largely ignored in the renal literature.

\section{Language Barriers: Issues with Translation and Interpretation}

There are several reasons for the scarcity of research into the psychological well-being of South Asian patients with ESRD. First, there are often language barriers which make it difficult for clinicians and researchers alike to explore the concerns and symptoms of patients, and may indeed lead to misinterpretations of patient complaints [38, 39]. Of course, one way to advance screening of depression symptoms would be to undertake the translation of validated instruments to meet the linguistic needs of the South Asian community within the UK. Translation is however not without its challenges. Hussain-Gambles et al. [40] propose that studies utilizing translation and interpretation are often expensive and time-consuming, making them less attractive for funding. Where translation and interpretation is available, accurate exploration of experiences may be limited by several factors, including culturespecific idioms of distress. Western idioms used to describe psychological distress may not readily translate into other languages. This may result in patients endorsing certain items less frequently because they cannot relate to the language used, which is not the same as being 'symptom free'. Krause [41] reported on a group of South Asians in Bedford, UK, originating from Punjab in India and described the occurrence of the 'sinking heart', a culturebound idiom used to describe specific somatic symptoms. Whilst these symptoms were similar to the Western conceptualization of stress, the 'sinking heart' was seen as a culture-bound 
syndrome that resulted from a combination of social, physical and emotional factors. Consequently, Bhui et al. [42] suggest that translators and interpreters must be equally competent in both the source and second language and understand the semantic range of both languages, whilst being sensitive to variations in cultural understandings of distress. Edwards [43] further suggests that translation can be facilitated by the translator being a native speaker in the target language and fluent in the language of the text to be translated.

There are also disparities in the quality of translation undertaken in studies where scales have been used across cultural and ethnic groups. Translation should be an iterative process, involving both forward and back translation to ensure face and content validity. However, there are marked differences in the use of forward- or backward-only translation with monoor bilingual tests of accuracy [44]. Poor translation methods are often accompanied by a lack of reporting on the validity of translations, with sensitivity and reliability analyses being undertaken without confidence in the conceptual equivalence of scales for use across cultures [42]. This results in the limitations of measures being advanced without providing confidence in the validity of the translated scales. Criterion validity, for example, cannot then be tested. Different constructions of psychological distress across cultures may well have clinical implications which undermine the precise assessment of conditions such as depression. Conducting and evaluating the process of translation needs to be the core aspect of the research design and reporting, without which it will be difficult for professionals to select appropriate instruments to screen for distress in different patient groups.

For a vast number of patients, translated scales, at least within their self-complete format, will not be suitable, and patients will require the use of interpreters in the completion of the scales. Feehally [45], for example, states that $25 \%$ of South Asians aged over 65 years living in Leicester, UK, cannot read or write in their native language or English. The use of interpreters to communicate with patients also requires a number of considerations. In a clinical setting, interpreters need to translate the patient's narrative promptly, leaving little time for considering emotional components of language, attitudes and beliefs, which play a vital role in informing clinical interpretations and treatment of symptoms. Furthermore, the presence of an interpreter may hinder the development of an empathic relationship between clinicians and patients, with the non-verbal aspects of communication often being lost when communicating through an interpreter [46]. There may also be variations in how interpreters view their roles, with research suggesting that for some, the role is related solely to accurate communication of information, whilst others view cultural advocacy, sensitivity and social support as fundamental components [47] and factors which influence the assessment of mood. It is also necessary to be aware of cultural tensions between patients and interpreters that influence willingness to disclose symptoms [46]. So whilst translation and interpretation services are undoubtedly useful in clinical settings, research is still lacking in specifying the optimal conditions for ensuring that vital aspects of communication are not lost or misinterpreted through such modes of communication with patients. Studies undertaking the task of translating screening instruments for use in the South Asian ESRD population need to pay due attention to the process of translation and on reporting key information that will allow professionals to make informed decisions in how best to assess the psychological requirements of patients.

\section{Conceptual Issues}

It is also highly plausible that there will be differences in the meaning attributed to symptoms of depression in cultures where there are no linguistic equivalents for the term [39]. Indeed, the way patients conceptualize their distress is predicative of not only symptom presentation [48], but also help seeking behaviour $[49,50]$. Whilst the beliefs of South Asians within the UK may be influenced by the host society, culture-specific beliefs are also important in understanding physical and mental illnesses. Several studies have found that although 
South Asians may be aware of the so-called psychosocial features of depression, they do not favour a biological model of mental illness, which is the core of the DSM-IV and ICD-10 criteria [51-55]. Furthermore, South Asians are more likely to see depression as the result of worry, whereas white Euro-Americans have been found to attribute depression to hopelessness and low mood [54].

The literature on mental health attributions in South Asians has consistently shown that 'spirituality', 'karma' and 'God's will' are often advanced to help explain medical and psychiatric illness $[39,53,56,57]$. In a clinical study of Canadian South Asian Punjabi-speaking women with breast cancer, Gurm et al. [58] found that patients predominantly made sense of illness in the context of spirituality. Spirituality provided meaning to illness, often in light of cancer being seen as part of a person's 'karma' or 'punishment' for sins. Nonetheless, spirituality offered a positive framework for coping through methods such as prayer. These findings have been replicated with other clinical groups including patients with coronary heart disease (CHD). South Asian patients with CHD encompass biological causes in their explanations of $\mathrm{CHD}$, but spiritual factors also form a core part of illness attributions [59]. More recently, a survey of South Asians in the United States found that whilst $88 \%$ of respondents favoured the belief that mental illness is a medical problem, there was stigma attached to seeking professional help, with South Asian patients preferring to turn to close family and friends for support [60]. Seeking help for psychiatric issues can be seen as a sign of weakness or as going against 'God's will', and openly disclosing illness may have broader social consequences for the family [61]. There may also be worry over being misunderstood by health professionals [50]. The meaning attributed to psychiatric symptoms thus varies across different cultural and ethnic groups, and many authors have expressed concern over the universal applicability of the Western model of mental conditions [62-64]. In practice, this means that screening instruments must capture culture-specific idioms of distress, and clinicians need to be aware of barriers that inhibit patients from freely disclosing their symptoms.

\section{Somatization}

A further challenge for clinicians in identifying psychiatric comorbidities pertains to differences in symptom presentation. Due to multiple cultural variants in the expression of distress, striking differences are found in symptom presentation cross-culturally. A topic that has attracted much attention across disciplines is the increased prevalence of somatic complaints amongst South Asians, which have often been labeled 'idioms' of emotional distress [39, 65-68]. Somatic complaints occur across all cultural groups, although there are marked differences in prevalence and features. Kirmayer and Young [69] caution against the belief that excess somatization in non-Western cultures represents an apparent 'primitiveness' or inability to express psychological distress. Psychological and somatic symptoms may be just as valid in the expression of distress, and the clinical significance of different symptoms remains unclear. For some cultural groups, expressing distress through physical complaints is likely to offer a socially acceptable route to disclosure. However, established scales such as the BDI-II and the Hospital Anxiety and Depression Scale [70] contain limited or no items related to the somatic features of psychological conditions [67]. This helpfully prevents the risk of items being endorsed due to an underlying medical reason but perhaps makes the scales less relevant for other cultural groups where somatic and psychological complaints coexist or where somatic complaints dominate and obscure psychological expressions of distress [67]. Indeed, Beliappa [66] proposes that in non-Western cultures where the mind and body are not viewed as distinct entities or compartmentalized, the distinction between psychological and somatic complaints may be less meaningful. The accurate screening and diagnosis of depression must therefore take account of cultural variations in both the conceptualization and expression of distress. 
Emic versus Etic Measures of Depression

Culture undoubtedly influences the expression of psychological distress, and health professionals are aware of the challenges in providing care for patients from diverse cultural and ethnic backgrounds [71]. In light of the issues related to differing conceptualizations, symptom presentation, and the complexities involved in utilizing translation and interpretation, it may seem sensible to employ culture-specific measures of depression, developed within the Indian subcontinent. Such a stance is perhaps best understood by considering the distinction made in cross-cultural research between the emic and etic approaches [72].

The emic approach focuses on culturally bound constructs and culture-specific measurement of a phenomenon. An emic measure would seek to capture the unique features of distress, as experienced by a particular ethnic group. For example, using this approach, researchers have identified culture-bound somatic complaints, which act as idioms of distress. Krause [41] identified the 'sinking heart' in Punjabis in Bedford, UK, which most closely resembled the Western conceptualization of stress. Similarly, Sulaiman et al. [73] reported the term 'broken body' used by an Arab community sample in Dubai to describe depressive phenomenon. Exploration of culture-bound syndromes has also led to the development of a number of indigenous instruments for the measurement of psychological distress. For example, the Primary Care Psychiatric Questionnaire [74] was developed to measure non-psychotic features of distress in India. There are numerous other examples of screening instruments developed locally to meet the linguistic needs and capture the unique features of distress, as experienced by members of a given ethnic group [67, 75, 76].

The etic approach, on the other hand, seeks to identify features of distress that are culture-general $[72,77]$. This approach assumes that the aetiology of distress is universal and emphasizes equivalence in the measurement of distress cross-culturally [78]. Such an approach is advanced by studies utilizing measures developed in the West, such as the BDI-II, with other ethnic groups.

At present, there is limited evidence to aid clinicians in making informed decisions about whether to employ an emic or etic approach to screen for distress in South Asian patients. Bhui et al. [79] describe a study in which the screening accuracy of the Amritsar Depression Inventory (ADI [80]), an instrument developed and validated in Punjab in India, and the General Health Questionnaire (GHQ-12 [81]) was compared against a clinical diagnostic interview for primary care attenders originating from Punjab in India. The study found that for Punjabis who had lived in the UK for over 30 years, the ADI was no better than chance at detecting depression symptoms, although the scale functioned as well as the GHQ-12 in English subjects. The GHQ-12 was more accurate overall in the detection of depression symptoms in English and Punjabi primary care attenders.

More recently, Stahl et al. [82] suggested that Western screening instruments such as the Centre for Epidemiological Studies Depression Scale (CES-D [83]) achieve sufficient sensitivity and specificity in identifying significant depressive symptoms in South Asian patients with diabetes in Singapore, although the results need to be interpreted with caution in light of the statistical power of the study. A review of depression screening instruments available in Urdu, on the other hand, concluded that there is insufficient evidence to advance the use of Western translated scales over measures developed in indigenous languages, primarily due to a lack of reporting on cross-cultural validity and criterion reliability [84].

Both emic (culture-specific) and etic (universal or culture-general) depression screening tools are currently employed across studies with both clinical and non-clinical groups of South Asians. However, many of the scales are employed without adequate testing of the validity of the scales in the population of interest, and indeed Bhui et al. [79] suggest that expressions of distress may vary between sub-groups of a broad ethnic group, especially 
due to the process of acculturation, i.e. the process through which members of a minority group adopt the beliefs and behaviours of the majority. Depression screening and diagnosis needs to be mindful of the complexities highlighted above both in the reliance on translation and interpretation, and the use of emic and etic screening instruments. For emic screening instruments, there needs to be careful consideration on how to assess the diagnostic accuracy of scales. The use of emic measures assumes that the experience of depression is culture-specific and so it may not, for example, be appropriate to compare such instruments with Western diagnostic criteria, such as those of the DSM-IV, which assume that depression is a universal phenomenon, the subjective experience of which is 'culturegeneral'.

\section{Conclusions}

The consequences of the under-representation of South Asian patients in research into the experience, measurement, diagnosis and treatment of depression in ESRD are farreaching, preventing benefits from being generalized across patient groups. Such practice also undermines the agenda of the UK National Health Service to reduce inequalities in health care provision. Depression screening and diagnostic schemes are limited by the assumption that depression, as defined by the Western biopsychiatric model, is a universal phenomenon, the subjective experience and expression of which is common across different cultural and ethnic groups. Renal health professionals need to be mindful of the issues outlined in this article when assessing a patient's psychological well-being. Information from interpreters should be used in conjunction with discussion with the patient's family and assessment from bilingual mental health professionals. Research into the thoughts and feelings of South Asian patients with ESRD, the evaluation of emic measures of psychological distress or the adaptation of etic scales is encouraged and should be based on sound evaluation of both the process of scale construction/translation and reporting on validity.

\section{References}

1 Cukor D, Rosenthal DS, Jindal RM, Brown CD, Kimmel PL: Depression is an important contributor to low medication adherence in hemodialyzed patients and transplant recipients. Kidney Int 2009; 75:1223-1229.

2 Chilcot J, Wellsted D, Da Silva-Gane M, Farrington K: Depression on dialysis. Nephron Clin Pract 2008;108:c256-c264.

3 Devins G, Hunsley J, Mandin H, Taub K, Paul L: The marital context of end-stage renal disease: Illness intrusiveness and perceived changes in family environment. Ann Behav Med 1997;19:325-332.

4 O'Brien M: Compliance behavior and long-term maintenance dialysis. Am J Kidney Dis 1990;15: 209-214.

5 Reiss D: Patient, family, and staff responses to end-stage renal disease. Am J Kidney Dis 1990;15: 194-200.

6 Karasz A, Garcia N, Ferri L: Conceptual models of depression in primary care patients: a comparative study. J Cross Cult Psychol 2009;40:1041-1059.

7 Steptoe A: Depression and Physical Illness. Cambridge, Cambridge University Press, 2006.

8 Smyth R: Depression in physical illness. J R Coll Physicians Edinb 2009;39:337-342.

9 Drayer RA, Piraino B, Reynolds Iii CF, Houck PR, Mazumdar S, Bernardini J, Shear MK, Rollman BL: Characteristics of depression in hemodialysis patients: symptoms, quality of life and mortality risk. Gen Hosp Psychiatry 2006;28:306-312.

10 Tsay SL, Healstead M: Self-care self-efficacy, depression, and quality of life among patients receiving hemodialysis in Taiwan. Int J Nurs Stud 2002;39:245-251. 
11 Chilcot J, Davenport A, Wellsted D, Firth J, Farrington K: An association between depressive symptoms and survival in incident dialysis patients. Nephrol Dial Transplant 2011;26:1628-1634.

12 Diefenthaeler EC, Wagner MrB, Poli-de-Figueiredo CE, Zimmermann PR, Saitovitch D: Is depression a risk factor for mortality in chronic hemodialysis patients? Rev Bras Psiquiatr 2008;30:99-103.

13 Kimmel PL: Psychosocial factors in adult end-stage renal disease patients treated with hemodialysis: correlates and outcomes. Am J Kidney Dis 2000;35:S132-S140.

14 Peterson R, Kimmel P, Sacks C, Mesquita M, Simmens S, Reiss D: Depression, perception of illness and mortality in patients with end-stage renal disease. Int J Psychiatry Med 1991;21:343-354.

15 Riezebos RK, Nauta KJ, Honig A, Dekker FW, Siegert CEH: The association of depressive symptoms with survival in a Dutch cohort of patients with end-stage renal disease. Nephrol Dial Transplant 2010;25:231-236.

16 Chilcot J, Norton S, Wellsted D, Almond M, Davenport A, Farrington K: A confirmatory factor analysis of the Beck Depression Inventory-II in end-stage renal disease patients. J Psychosom Res 2011;71:148-153.

17 Kimmel P: Psychosocial factors in dialysis patients. Kidney Int 2001;59:1599-1613.

18 Koenig H, George L, Peterson B, Pieper C: Depression in medically ill hospitalized older adults: prevalence, characteristics, and course of symptoms according to six diagnostic schemes. Am J Psychiatry 1997;154:1376-1383.

19 Beck A, Steer R, Brown G: Beck Depression Inventory - 2nd Edition Manual. San Antonio, the Psychological Corporation, 1996.

20 Hada R, Khakurel S, Agrawal R, Kafle R, Bajracharya S, Raut K: Incidence of end stage renal disease on renal replacement therapy in Nepal. Kathmandu Univ Med J 2009;7:302-305.

21 Buck K, Feehally J: Diabetes and renal failure in Indo-Asians in the UK - a paradigm for the study of disease susceptibility. Nephrol Dial Transplant 1997;12:1555-1557.

22 Trehan A, Winterbottom J, Lane B, Foley R, Venning M, Coward R, MacLeod AM, Gokal R: Endstage renal disease in Indo-Asians in the North-West of England. QJM 2003;96:499-504.

23 Lightstone L, Rees AJ, Tomson C, Walls J, Winearls CG, Feehally J: High incidence of end-stage renal disease in Indo-Asians in the UK. QJM 1995;88:191-195.

24 Roderick PJ, Jones I, Raleigh VS, McGeown M, Mallick N: Population need for renal replacement therapy in Thames regions: ethnic dimension. BMJ 1994;309:1111-1114.

25 Burden AC, McNally PC, Feehally J, Walls J: Increased incidence of end-stage renal failure secondary to diabetes mellitus in Asian ethnic groups in the United Kingdom. Diabet Med 1992;9:641-645.

26 Roderick PJ, Raleigh VS, Hallam L, Mallick NP: The need and demand for renal replacement therapy in ethnic minorities in England. J Epidemiol Community Health 1996;50:334-339.

27 UK Renal Registry Report 2000. Bristol, UK Renal Registry.

28 Karter AJ, Ferrara A, Liu JY, Moffet HH, Ackerson LM, Selby JV: Ethnic disparities in diabetic complications in an insured population. JAMA 2002;287:2519-2527.

29 Ramachandran A, Snehalatha C, Latha E, Manoharan M, Vijay V: Impacts of urbanisation on the lifestyle and on the prevalence of diabetes in native Asian Indian population. Diabetes Res Clin Pract 1999;44:207-213.

30 Windmill DC, Jain N, Inston NG, Ready AR: Impact of a 'direct approach' to live kidney donation in the British Indo-Asian community. Transplant Proc 2005;37:551-552.

31 Roderick P, Byrne C, Casula A, Steenkamp R, Ansell D, Burden R, Nitsch D, Feest T: Survival of patients from South Asian and Black populations starting renal replacement therapy in England and Wales. Nephrol Dial Transplant 2009;24:3774-3782.

32 Pei Y, Greenwood C, Chery A, Wu G: Racial differences in survival of patients on dialysis. Kidney Int 2000;58:1293-1299.

33 Tonelli M, Hemmelgarn B, Gill J, Chou S, Culleton B, Klarenbach S, Manns B, Wiebe N, Gourishankar S: Patient and allograft survival of Indo Asian and East Asian dialysis patients treated in Canada. Kidney Int 2007;72:499-504.

34 Prasad S, Singh S, Duncan N, Cairns T, Griffith M, Hakim N, McLean A, Palmer A, Papaloi V, Taube D: Ethnicity and survival on dialysis in west London. Kidney Int 2004;66:2416-2421.

35 Mahajan S, Tiwari S, Kalra V, Masih J, Bhowmik D, Bansal R, Agarwal S: Analysis of depression and its effect on outcome among adult Indian peritoneal dialysis patients. Perit Dial Int 2007;27:94-96.

36 Anees M, Hameed F, Mumtaz A, Ibrahim M, Khan M: Dialysis-related factors affecting quality of life in patients on hemodialysis. Iran J Kidney Dis 2011;5:9-14. 
37 Bakewell A, Higgins R, Edmunds M: Quality of life in peritoneal dialysis patients: decline over time and association with clinical outcomes. Kidney Int 2002;61:239-248.

38 Conrad MM, Pacquiao DF: Manifestation, attribution, and coping with depression among Asian Indians from the perspectives of health care practitioners. J Transcult Nurs 2005;16:32-40.

39 Hussain F, Cochrane R: Depression in South Asian women living in the UK: a review of the literature with implications for service provision. Transcult Psychiatry 2004;41:253-270.

40 Hussain-Gambles M, Atkin K, Leese B: Why ethnic minority groups are under-represented in clinical trials: a review of the literature. Health Soc Care Community 2004;12:382-388.

41 Krause IB: Sinking heart: a Punjabi communication of distress. Soc Sci Med 1989;29:563-575.

42 Bhui K, Mohamud S, Warfa N, Craig TJ, Stansfeld SA: Cultural adaptation of mental health measures: improving the quality of clinical practice and research. Br J Psychiatry 2003;183:184-186.

43 Edwards N: Translating written material for community health research and service delivery: guidelines to enhance the process. Can J Public Health 1994;85:67-70.

44 Maneesriwongul W, Dixon JK: Instrument translation process: a methods review. J Adv Nurs 2004; 48:175-186.

45 Feehally J: Ethnicity and renal disease: questions and challenges. Clin Med 2003;3:578-582.

46 Pugh MA, Vetere A: Lost in translation: an interpretative phenomenological analysis of mental health professionals' experiences of empathy in clinical work with an interpreter. Psychol Psychother 2009;82:305-321.

47 Butow P, Lobb E, Jefford M, Goldstein D, Eisenbruch M, Girgis A, King M, Sze M, Aldridge L, Schofield P: A bridge between cultures: interpreters' perspectives of consultations with migrant oncology patients. Support Care Cancer 2010, E-pub ahead of print.

48 Raguram R, Weiss MG, Channabasavanna SM, Devins GM: Stigma, depression, and somatization in South India. Am J Psychiatry 1996;153:1043-1049.

49 Sheikh S, Furnham A: A cross-cultural study of mental health beliefs and attitudes towards seeking professional help. Soc Psychiatry Psychiatr Epidemiol 2000;35:326-334.

50 Loewenthal KM, Cinnirella M: Beliefs about the efficacy of religious, medical and psychotherapeutic interventions for depression and schizophrenia among women from different cultural-religious groups in Great Britain. Transcult Psychiatry 1999;36:491-504.

51 Karasz A: Cultural differences in conceptual models of depression. Soc Sci Med 2005;60:1625-1635.

52 Pereira B, Andrew G, Pednekar S, Pai R, Pelto P, Patel V: The explanatory models of depression in low income countries: listening to women in India. J Affect Disord 2007;102:209-218.

53 Hussain F, Cochrane R: Depression in South Asian women: Asian women's beliefs on causes and cures. Ment Health Relig Cult 2002;5:285-311.

54 Lawrence V, Murray J, Banerjee S, Turner S, Sangha K, Byng R, Bhugra D, Huxley P, Tylee A, Macdonald A: Concepts and causation of depression: a cross-cultural study of the beliefs of older adults. Gerontologist 2006;46:23-32.

55 Fenton S, Sadiq-Sangster A: Culture, relativism and the expression of mental distress: South Asian women in Britain. Sociol Health Illn 1996;18:66-85.

56 Yamey G, Greenwood R: Religious views of the 'medical' rehabilitation model: a pilot qualitative study. Disabil Rehabil 2004;26:455-462.

57 Johnson JL, Bottorff JL, Balneaves LG, Grewal S, Bhagat R, Hilton BA, Clarke H: South Asian women's views on the causes of breast cancer: images and explanations. Patient Educ Couns 1999;37: 243-254.

58 Gurm BK, Stephen J, MacKenzie G, Doll R, Barroetavena MC, Cadell S: Understanding Canadian Punjabi-speaking South Asian women's experience of breast cancer: a qualitative study. Int J Nurs Stud 2008;45:266-276.

59 Tirodkar MA, Baker DW, Khurana N, Makoul G, Paracha MW, Kandula NR: Explanatory models of coronary heart disease among South Asian immigrants. Patient Educ Couns 2011;85:230-236.

60 Rao V, Goga J, Inscore A, Kosi R, Khushalani S, Rastogi P, Subramaniam G, Jayaram G: Attitudes towards mental illness and help-seeking behaviors among South Asian Americans: results of a pilot study. Asian J Psychiatr 2011;4:76.

61 Pilkington A, Msetfi RM, Watson R: Factors affecting intention to access psychological services amongst British Muslims of South Asian origin. Ment Health Relig Cult 2011, E-pub ahead of print.

62 Aisenberg E: Evidence-based practice in mental health care to ethnic minority communities: has its practice fallen short of its evidence? Soc Work 2008;53:297-306. 
63 Summerfield D: How scientifically valid is the knowledge base of global mental health? BMJ 2008; 336:992-994.

64 Canino G, Alegría M: Psychiatric diagnosis - is it universal or relative to culture? J Child Psychol Psychiatry 2008;49:237-250.

65 Bhui K, Bhugra D, Goldberg D, Sauer J, Tylee A: Assessing the prevalence of depression in Punjabi and English primary care attenders: the role of culture, physical illness and somatic symptoms. Transcult Psychiatry 2004;41:307-322.

66 Beliappa J: Illness or Distress? Alternative Models of Mental Health. London, Confederation of Indian Organisations, 1991.

67 Mumford DB, Bavington JT, Bhatnagar KS, Hussain Y, Mirza S, Naraghi MM: The Bradford Somatic Inventory. A multi-ethnic inventory of somatic symptoms reported by anxious and depressed patients in Britain and the Indo-Pakistan subcontinent. Br J Psychiatry 1991;158:379-386.

68 Wilson M, MacCarthy B: GP consultation as a factor in the low rate of mental health service use by Asians. Psychol Med 1994;24:113-119.

69 Kirmayer LJ, Young A: Culture and somatization: clinical, epidemiological, and ethnographic perspectives. Psychosom Med 1998;60:420-430.

70 Zigmond AS, Snaith RP: The hospital anxiety and depression scale. Acta Psychiatr Scand 1983;67: 361-370.

71 Bhui K, Warfa N, Edonya P, McKenzie K, Bhugra D: Cultural competence in mental health care: a review of model evaluations. BMC Health Serv Res 2007;31:7-15.

72 Brislin RW, Lonner WJ, Thorndike RM: Crosscultural Research Methods. New York, Wiley, 1973.

73 Sulaiman SOY, Bhugra D, de Silva P: Perceptions of depression in a community sample in Dubai. Transcult Psychiatry 2001;38:201-218.

74 Srinivasan T, Suresh T: Non-specific symptoms and screening of non-psychotic morbidity in primary care. Indian J Psychiatry 1990;32:77-82.

75 Abas MA, Phillips C, Carter J, Walter J, Banerjee S, Levy R: Culturally sensitive validation of screening questionnaires for depression in older African-Caribbean people living in south London. Br J Psychiatry 1998;173:249-254.

76 Patel V, Simunyu E, Gwanzura F, Lewis G, Mann A: The Shona Symptom Questionnaire: the development of an indigenous measure of common mental disorders in Harare. Acta Psychiatr Scand 1997;95:469-475.

77 Leong FT, Leung K, Cheung FM: Integrating cross-cultural psychology research methods into ethnic minority psychology. Cultur Divers Minor Psychol 2010;16:590-597.

78 Cheng AT: Case definition and culture: are people all the same? Br J Psychiatry 2001;179:1-3.

79 Bhui K, Bhugra D, Goldberg D: Cross-cultural validity of the Amritsar Depression Inventory and the General Health Questionnaire amongst English and Punjabi primary care attenders. Soc Psychiatry Psychiatr Epidemiol 2000;35:248-254.

80 Singh G, Verma H, Verma R, Kaur H: A new depressive inventory. Indian JPsychiatry 1974;16:83-188.

81 Goldberg D, Gater R, Sartorius N, Ustun T, Piccinelli M, Gureje O, Rutter C: The validity of the two versions of the GHQ in the WHO study of mental illness in general health care. Psychol Med 1997; 27:191-197.

82 Stahl D, Sum CF, Lum SS, Liow PH, Chan YH, Verma S, Chua HC, Chong SA: Screening for depressive symptoms: validation of the center for epidemiologic studies depression scale (CES-D) in a multiethnic group of patients with diabetes in Singapore. Diabetes Care 2008;31:1118-1119.

83 Radloff L: The CES-D scale: a self-report depression scale for research in the general population. Appl Psychol Meas 1977;1:3.

84 Ahmer S, Faruqui R, Aijaz A: Psychiatric rating scales in Urdu: a systematic review. BMC Psychiatry 2007;7:59. 